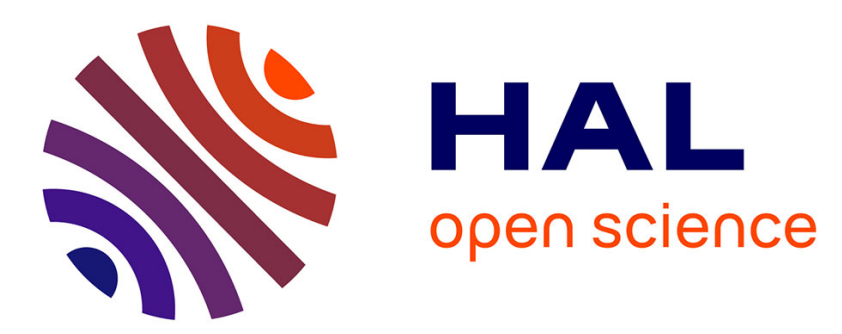

\title{
Imaging linear polarimetry using a single ferroelectric liquid crystal modulator
}

Luc Gendre, Alban Foulonneau, Laurent Bigue

\section{To cite this version:}

Luc Gendre, Alban Foulonneau, Laurent Bigue. Imaging linear polarimetry using a single ferroelectric liquid crystal modulator. Applied optics, 2010, 49 (25), pp.4687-4699. 10.1364/AO.49.004687 . hal00828109

\section{HAL Id: hal-00828109 \\ https://hal.science/hal-00828109}

Submitted on 30 May 2013

HAL is a multi-disciplinary open access archive for the deposit and dissemination of scientific research documents, whether they are published or not. The documents may come from teaching and research institutions in France or abroad, or from public or private research centers.
L'archive ouverte pluridisciplinaire HAL, est destinée au dépôt et à la diffusion de documents scientifiques de niveau recherche, publiés ou non, émanant des établissements d'enseignement et de recherche français ou étrangers, des laboratoires publics ou privés. 


\title{
Imaging linear polarimetry using a single ferroelectric liquid crystal modulator
}

\author{
Luc Gendre, ${ }^{*}$ Alban Foulonneau, and Laurent Bigué \\ Laboratoire Modélisation Intelligence Processus Systèmes (EA 2332), Ecole Nationale Supérieure d'Ingénieurs \\ Sud Alsace, Université de Haute-Alsace, 12 rue des Frères Lumière, 68093 Mulhouse Cedex, France \\ ${ }^{*}$ Corresponding author: luc.gendre@ uha.fr
}

Received 29 March 2010; revised 23 July 2010; accepted 23 July 2010;

posted 2 August 2010 (Doc. ID 126203); published 24 August 2010

\begin{abstract}
In the field of polarimetry, ferroelectric liquid crystal cells are mostly used as bistable polarization rotators suitable to analyze crossed polarizations. This paper shows that, provided such a cell is used at its nominal wavelength and correctly driven, its behavior is close to that of a tunable half-wave plate, and it can be used with much benefit in lightweight imaging polarimetric setups. A partial Stokes polarimeter using a single digital video camera and a single ferroelectric liquid crystal modulator is designed and implemented for linear polarization analysis. Polarization azimuthal angle and degree of linear polarization are available at 150 frames per second with a good accuracy. (C) 2010 Optical Society of America OCIS codes: $\quad 110.5405,120.5410,160.3710,160.2260,230.4110$.
\end{abstract}

\section{Introduction}

Imaging of polarization is useful in remote sensing [1], military applications [2], and astronomy [3]; additionally, it serves as an aid to medical diagnosis [4]. Polarimetry principles have been known for one and a half century [5], but designing a dynamic polarimeter that allows the polarization to be analyzed as fast as possible remains an issue. In the beginning, polarization was analyzed by mechanically rotating polarizing plates. This kind of architecture has reached a high level of accuracy, and nowadays the issue of analysis speed has become increasingly important, especially for imaging applications of dynamic phenomena. Basics of imaging polarimetry appeared in the literature in 1981 [6] and, since then, different techniques have been developed for imaging analysis of polarization. They either rely on division of amplitude/aperture [7-9], wavefront [10,11], or time [12]. From the optical point of view, division of time architectures lead to the simplest implementations and they prove very efficient when the mechanically rotated elements are replaced by electronically tunable

$0003-6935 / 10 / 254687-13 \$ 15.00 / 0$

(C) 2010 Optical Society of America liquid crystal cells [3, $\underline{3}]$. For instance, liquid crystal cells allow acquisition speeds that are compatible with video frame rates. Wolff et al. [13] proposed in 1997 the use of nematic liquid crystal devices for polarimetry. Their implementation was able to capture polarization information at up to 50 frames per second (fps). A higher speed can be obtained with ferroelectric liquid crystal cells. Gandorfer [3] reached $250 \mathrm{fps}$, while Jaulin et al. $[\underline{14}, \underline{15}]$ conducted experiments at up to $360 \mathrm{fps}$. Up to now, all the liquid crystal implementations providing at least three Stokes parameters used either a single nematic device running slowly, or two ferroelectric devices able to reach high speeds.

In this paper, a prototype portable polarimeter using a single ferroelectric liquid crystal cell in a division of time architecture is presented. It is composed of a ferroelectric liquid crystal (LC) modulator in front of a commercial digital video camera. It provides all Stokes information about the linearly polarized components of light. To do so, the basic idea consists in using the ferroelectric liquid crystal cell with an adjustable control, whereas it is primarily designed to respond to a bistable control. The experiments showed that the LC cell's behavior was close to that of a half-wave plate but, to improve the system 
performance, using an experimental numerical model was preferred.

Section 2 introduces the basics of polarization used in this paper. Section 3 focuses on the scheme used and Section 4 details the characterization of the system. Section 5 presents validation results, and a quick discussion is exposed Section $\underline{6}$.

\section{Polarization Formalism}

\section{A. Stokes Vector: a Light Polarization State Representation}

Polarization of light is represented by a vector [5], called the Stokes vector, that allows representation of totally polarized light, unpolarized light, as well as partially polarized light. The general expression of this vector is $\mathbf{S}=\left[\begin{array}{llll}s_{0} & s_{1} & s_{2} & s_{3}\end{array}\right]^{t}$, where $s_{0}$ denotes the total intensity, $s_{1}$ is the excess parallel to perpendicular light, and $s_{2}$ and $s_{3}$ define nature and handedness of the wave, respectively.

In this paper, we focus on the evaluation of the first three components of the Stokes vector, i.e., those that represent the linear polarization. Therefore, the circular polarization component $s_{3}$ is ignored. This does not mean this component to be nil, but rather in our treatment $s_{3}$ remains unknown and is considered as unpolarized light with regards to the linear polarization. Thus, the partial Stokes vector considered is $\mathbf{S}=\left[\begin{array}{llll}s_{0} & s_{1} & s_{2} & -\end{array}\right]^{t}$.

From this vector can be derived the degree of linear polarization (DOLP) and the azimuthal angle $\psi[\underline{16]}$ :

$$
\begin{gathered}
\operatorname{DOLP}=\sqrt{s_{1}^{2}+s_{2}^{2}} / s_{0}, \\
\tan (2 \psi)=\frac{s_{2}}{s_{1}} .
\end{gathered}
$$

The DOLP represents the fraction of the intensity attributable to linearly polarized light states[1]. The DOLP varies from 0 for unpolarized light to 1 for totally linearly polarized light; intermediate values indicate partially linearly polarized light. The azimuthal angle is the angle of the major axis of the polarization ellipse with respect to the angular origin, which is set to be the horizontal in this paper.

Estimation of this partial Stokes vector is sufficient to analyze the linear polarization of the light coming from a scene, for example, evaluation of linear depolarization [17], discrimination between materials $[18,19]$, or analysis of painted surfaces [20].

\section{B. Mueller Matrix: a Polarimetric Transfer Function}

Analyzing a Stokes vector $\mathbf{S}_{\text {out }}$ gives information on the light polarization coming from a scene. Provided the Stokes vector $\mathbf{S}_{\text {in }}$ of the light coming toward the scene is known and controllable, a characterization of each element in the scene becomes possible. A transfer function, called the Mueller matrix $\mathbf{M}$, can be determined for each element. This $4 \times 4$ matrix is defined by the following equation:

$$
\mathbf{S}_{\text {out }}=\mathbf{M} \cdot \mathbf{S}_{\text {in }} \text {. }
$$

Common elements in polarimetry (polarizers, retarders) have a well-known Mueller matrix to model their polarimetric response. In this paper, we are using linear polarizers and half-wave plates. The general expressions of the Mueller matrices of these two components for a respective orientation angle $\beta$ and $\theta$ of their fast axis, with respect to the angular origin, are

$$
\begin{gathered}
\mathbf{M}_{\mathrm{Pol}}(\beta) \\
=\frac{1}{2}\left[\begin{array}{cccc}
1 & \cos (2 \beta) & \sin (2 \beta) & 0 \\
\cos (2 \beta) & \cos ^{2}(2 \beta) & \cos (2 \beta) \sin (2 \beta) & 0 \\
\sin (2 \beta) & \cos (2 \beta) \sin (2 \beta) & \sin ^{2}(2 \beta) & 0 \\
0 & 0 & 0 & 0
\end{array}\right] \\
\mathbf{M}_{\frac{\bar{\alpha}}{2}}(\theta)=\left[\begin{array}{cccc}
1 & 0 & 0 & 0 \\
0 & \cos (4 \theta) & \sin (4 \theta) & 0 \\
0 & \sin (4 \theta) & -\cos (4 \theta) & 0 \\
0 & 0 & 0 & -1
\end{array}\right]
\end{gathered}
$$

\section{Polarization Analysis Model}

Our liquid crystal modulator is manufactured by Boulder Nonlinear Systems, Inc. (BNS)[21], and belongs to the MS Series. It is composed of a ferroelectric liquid crystal (FLC) cell in front of a linear polarizer. With the electronic driver provided by the manufacturer, the modulator is supposed to act as a bistable polarization rotator at $633 \mathrm{~nm}$, with fast axis switching from $0^{\circ}$ to $45^{\circ}$. No other configuration is available with this electronic driver. Recent experiments conducted with a rather similar device using an improved electronic driver [22] let us think that the FLC cell can act as a tunable half-wave plate. As a consequence, the modulator can theoretically be used as a polarization state analyzer (PSA) at $633 \mathrm{~nm}$ to analyze linear polarization, provided the electrical control is correctly designed. Because the present work aims to experimentally confirm that the modulator can effectively be used as a PSA, any further modeling or experimentation is done at $633 \mathrm{~nm}$, and the FLC cell is assumed during preliminary experiments to be a half-wave plate whose orientation varies as a function of the applied voltage.

\section{A. PSA Model}

The polarization orientation reference is that of the linear polarizer of the modulator. This polarizer is oriented horizontally and remains static. Considering an incident Stokes vector $\mathbf{S}_{\text {in }}=$ $\left(\begin{array}{llll}s_{\text {in } 0} & s_{\text {in } 1} & s_{\text {in } 2} & s_{\text {in } 3}\end{array}\right)^{t}$ and combining Eqs. (3) - ( $\left.\underline{5}\right)$, the intensity $I(\theta)$ that would be measured by a 
photodetector placed after the modulator is

$$
I(\theta)=s_{\text {out } 0}(\theta)=\frac{1}{2}\left[s_{\text {in } 0}+s_{\text {in } 1} \cdot \cos (4 \theta)+s_{\text {in } 2} \cdot \sin (4 \theta)\right]
$$

By measuring three different intensities $I_{1}, I_{2}$, and $I_{3}$, corresponding to three different angles $\left(\theta_{1}, \theta_{2}, \theta_{3}\right)$, the following system can be defined:

$$
\left[\begin{array}{l}
I_{1} \\
I_{2} \\
I_{3}
\end{array}\right]=\mathbf{A}\left[\begin{array}{c}
s_{\text {in } 0} \\
s_{\text {in } 1} \\
s_{\text {in } 2}
\end{array}\right]
$$

where

$$
\mathbf{A}=\frac{1}{2}\left[\begin{array}{ccc}
1 & \cos \left(4 \theta_{1}\right) & \sin \left(4 \theta_{1}\right) \\
1 & \cos \left(4 \theta_{2}\right) & \sin \left(4 \theta_{2}\right) \\
1 & \cos \left(4 \theta_{3}\right) & \sin \left(4 \theta_{3}\right)
\end{array}\right]
$$

A is called the system matrix [23].

Provided the matrix A is rank 3 , that is, if the three angles are not equal modulo $\pi / 2$, and calling $\mathbf{A}^{-1}$ the inverse, it is possible to determine the three parameters $s_{\mathrm{in} 0}, s_{\mathrm{in} 1}$, and $s_{\mathrm{in} 2}$ using the following equation:

$$
\left[\begin{array}{c}
s_{\text {in } 0} \\
s_{\text {in } 1} \\
s_{\text {in } 2}
\end{array}\right]=\mathbf{A}^{-1}\left[\begin{array}{c}
I_{1} \\
I_{2} \\
I_{3}
\end{array}\right]
$$

The matrix $\mathbf{A}^{-1}$ is the data reduction matrix (DRM) [23] of our PSA.

In practice, to improve the determination of the Stokes vector S, a larger number of intensity measurements $N$ can be performed [24], and the pseudoinverse of the $N \times 3$ matrix A can be considered instead of the inverse. But in this paper, to minimize experimental issues, only three intensity measurements are performed.

In the following work, the polarizing element consists of a liquid crystal cell, whose depolarization capabilities may not be neglected. If an isotropic depolarization is assumed as

$$
\mathbf{M}_{\Delta}=\left[\begin{array}{llll}
1 & 0 & 0 & 0 \\
0 & d & 0 & 0 \\
0 & 0 & d & 0 \\
0 & 0 & 0 & d
\end{array}\right]
$$

then Eq. (ㅁ) is written as

$$
\mathbf{M}_{\frac{\lambda}{2}}(\theta)=\left[\begin{array}{cccc}
1 & 0 & 0 & 0 \\
0 & d \cos (4 \theta) & d \sin (4 \theta) & 0 \\
0 & d \sin (4 \theta) & -d \cos (4 \theta) & 0 \\
0 & 0 & 0 & -d
\end{array}\right],
$$

and Eq. (6) is written as

$$
\begin{aligned}
I(\theta) & =s_{\text {out } 0}(\theta) \\
& =\frac{1}{2}\left[s_{\mathrm{in} 0}+s_{\mathrm{in} 1} \cdot d \cdot \cos (4 \theta)+s_{\mathrm{in} 2} \cdot d \cdot \sin (4 \theta)\right],
\end{aligned}
$$

which leads to the following system matrix:

$$
\mathbf{A}=\frac{1}{2}\left[\begin{array}{lll}
1 & d \cos \left(4 \theta_{1}\right) & d \sin \left(4 \theta_{1}\right) \\
1 & d \cos \left(4 \theta_{2}\right) & d \sin \left(4 \theta_{2}\right) \\
1 & d \cos \left(4 \theta_{3}\right) & d \sin \left(4 \theta_{3}\right)
\end{array}\right]
$$

\section{B. $\theta$ Angle Identification Method}

To choose the three angles $\theta_{1}, \theta_{2}$, and $\theta_{3}$, the modulator characteristic representing the orientation angle $\theta$ of the equivalent half-wave plate versus the voltage $V$ applied to the FLC cell has to be established. Therefore, the angle should be referred to as $\theta(V)$; however, because there is no possible ambiguity, hereafter, it is simply called $\theta$.

To do so, for each voltage $V$, the four following Stokes vectors listed in Eq. (14) are generated. Each of them represents a linear polarization oriented horizontally, vertically, at $45^{\circ}$, and at $135^{\circ}$, respectively.

$$
\left\{\mathbf{S}_{\mathbf{h}}, \mathbf{S}_{\mathbf{v}}, \mathbf{S}_{45}, \mathbf{S}_{135}\right\}=\left\{\left[\begin{array}{l}
1 \\
1 \\
0 \\
0
\end{array}\right],\left[\begin{array}{c}
1 \\
-1 \\
0 \\
0
\end{array}\right],\left[\begin{array}{l}
1 \\
0 \\
1 \\
0
\end{array}\right],\left[\begin{array}{c}
1 \\
0 \\
-1 \\
0
\end{array}\right]\right\}
$$

For each vector, the intensity of the light coming out of the modulator (FLC cell and polarizer) is measured. The following system is then established using Eq. (12), assuming the FLC modulator is a half-wave plate with an azimuthal angle $\theta$ and an isotropic depolarization $d$ :

$$
\left\{\begin{array}{cc}
I_{h}=\frac{1}{2}[1+d \cos (4 \theta)] & \text { for } \mathbf{S}_{\mathbf{h}} \text { in input } \\
I_{v}=\frac{1}{2}[1-d \cos (4 \theta)] & \text { for } \mathbf{S}_{\mathbf{v}} \text { in input } \\
I_{45}=\frac{1}{2}[1+d \sin (4 \theta)] & \text { for } \mathbf{S}_{\mathbf{4 5}} \text { in input } \\
I_{135}=\frac{1}{2}[1-d \sin (4 \theta)] & \text { for } \mathbf{S}_{\mathbf{1 3 5}} \text { in input }
\end{array}\right.
$$

where each intensity represents the measured intensity normalized by the maximum intensity of the incident light.

Thus,

$$
\begin{cases}\theta=\frac{1}{4} \arctan \left(\frac{I_{45}-I_{135}}{I_{h}-I_{v}}\right) & \text { for } I_{h} \neq I_{v} \\ \theta=+22.5^{\circ} & \text { for } I_{h}=I_{v} \text { and } I_{135}=0 \\ \theta=-22.5^{\circ} & \text { for } I_{h}=I_{v} \text { and } I_{45}=0\end{cases}
$$

and

$$
d=\sqrt{\left(I_{h}-I_{v}\right)^{2}+\left(I_{45}-I_{135}\right)^{2}} .
$$


Using Eq. (16) for the whole range of electrical control voltages, the characteristic curve $\theta(V)$ can be determined. Then, three specific voltages $V_{1}, V_{2}$, and $V_{3}$, corresponding to angles $\theta_{1}, \theta_{2}$, and $\theta_{3}$, can be chosen to compute the DRM A ${ }^{-1}$ as detailed below.

\section{Experimental Characterization and Implementation}

\section{A. Experimental Setup}

A scheme and a picture of the polarimeter is presented Fig. 1. The modulator is composed of an FLC cell and a linear polarizer. The optical setup used for characterization is presented Fig. 2. Although the modulator is intended to be used for imaging applications, the characterization is done with spatially averaged signals. An intensity stabilized $\mathrm{He}-\mathrm{Ne}$ laser followed by a beam expander $\left(\mathrm{MO}, \mathrm{PH}\right.$, and $\left.L_{1}\right)$ generates an enlarged beam at $633 \mathrm{~nm}$, wavelength for which the FLC cell is supposed to act as a halfwave plate. A static linear polarizer $\left(P_{1}\right)$ oriented at $0^{\circ}$ guarantees a constant polarization orientation input to the half-wave plate $(\lambda / 2)$. Mechanically rotating this half-wave plate generates the four different Stokes vectors expressed in Eq. (14) used as successive inputs. The polarization of the light outgoing from the FLC cell is analyzed by a static linear polarizer $\left(P_{2}\right)$ oriented at $0^{\circ}$, and the light is focused with a lens $\left(L_{2}\right)$ on a photodetector. The set (FLC cell, $P_{2}$ ) is the PSA to be characterized. As shown Fig. 1, an interferential filter centered around $633 \mathrm{~nm}$ is used to allow for outdoor operations with uncontrolled lighting conditions. Even though, for this paper, the work was done under controlled illumination, for technical reasons, the interferential filter is actually included in the setup, whereas it has not been represented Fig. 2. In the following, temperature, which is known to play an important role in LC behavior [25], was monitored but not controlled. Temperature changes remained within $+/-0.5 \mathrm{~K}$ for the whole work and exhibit little influence on our experimental data. The average room temperature was $20.3{ }^{\circ} \mathrm{C}$.

\section{B. Intensity Measurements and Angle Estimation}

For each of the four Stokes vectors $\mathbf{S}_{\mathrm{h}}, \mathbf{S}_{\mathbf{v}}, \mathbf{S}_{45}$, and $\mathbf{S}_{\mathbf{1 3 5}}$, the modulator control voltage varies from $-5 \mathrm{~V}$ to $+5 \mathrm{~V}$. Using Eq. (16), the orientation angle

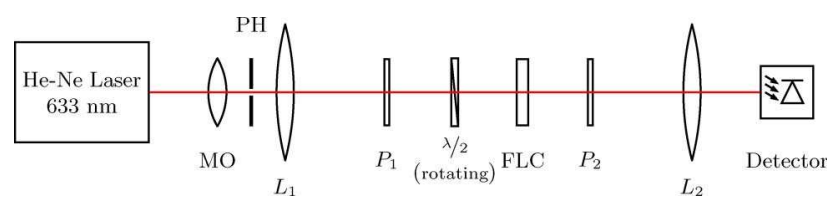

Fig. 2. (Color online) Setup for partial Mueller characterization of the FLC device. Microscope objective (MO), Pin Hole (PH), Lenses $\left(L_{1}\right.$ and $\left.L_{2}\right)$, static linear polarizers oriented at $0^{\circ}\left(P_{1}\right.$ and $\left.P_{2}\right)$, rotating half-wave plate $(\lambda / 2)$, ferroelectric liquid crystal cell (FLC).

of the half-wave plate modeling the FLC cell in function of the control voltage is determined. This process is repeated five times to get mean values, but figure dispersion proved to be very low.

Figure 3 reports the mean intensity measurements versus voltage, whereas the characteristic $\theta(V)$ deduced from these intensities is shown in Fig. 4. If the polarization could be analyzed at any angle from $0^{\circ}$ to $180^{\circ}$, it has been shown that the optimal configuration would correspond to equally spaced orientations, that is at $0^{\circ}, 60^{\circ}$, and $120^{\circ}[26]$, which correspond to angles for an equivalent halfwave plate of $0^{\circ}, 30^{\circ}$, and $60^{\circ}$. Unfortunately, Fig. 4 shows that such angles are not achievable if the control voltage stays within $\pm 5 \mathrm{~V}$ as suggested by the modulator manufacturer. Therefore, a suboptimal configuration has to be used and the extremal values $\theta(+5 \mathrm{~V})=0.6^{\circ}$ and $\theta(-5 \mathrm{~V})=41.5^{\circ}$ are chosen. The last angle could be chosen in the middle of the theoretical range of $\left[0^{\circ}, 45^{\circ}\right]$, that is at $22.5^{\circ}$, which corresponds to a control voltage of $+0.1 \mathrm{~V}$. However, choosing a control voltage anywhere in the whole amplitude range $[-5 \mathrm{~V},+5 \mathrm{~V}]$ may not be suitable. Figure 5 reports the sum of intensities $I_{h}+I_{v}$, which should $\bar{b}$ e constant all over the amplitude range (i.e., all over the angular range) if the FLC cell was a perfect half-wave plate. The modulator clearly departs from an ideal half-wave plate model between 0 and $+1 \mathrm{~V}$. Considering $I_{45}+I_{135}$ instead of $I_{h}+I_{v}$ leads to the same conclusions.

Finally, the third voltage is chosen as $-0.1 \mathrm{~V}$, which is outside the problematic range because the corresponding angle $31.2^{\circ}$ lies far enough from extremal values. Therefore, the DRM is evaluated using angles $41.5^{\circ}, 31.2^{\circ}$, and $0.6^{\circ}$, corresponding, respectively, to FLC cell control voltages $-5 \mathrm{~V},-0.1 \mathrm{~V}$, and $+5 \mathrm{~V}$.
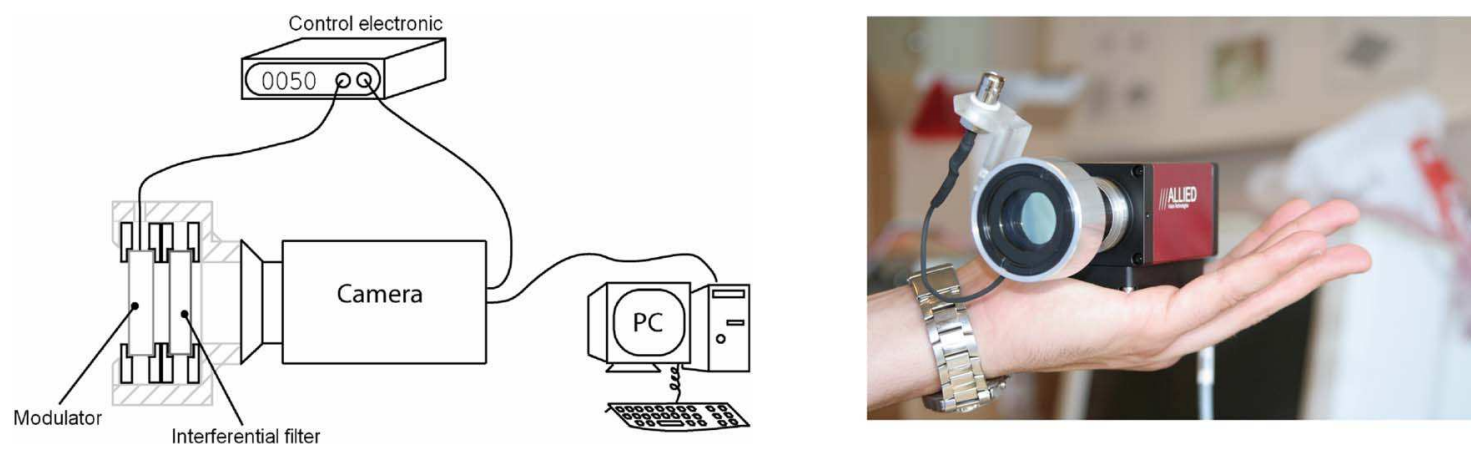

Fig. 1. (Color online) Polarimeter scheme and photo. The modulator is composed of an FLC cell and a linear polarizer. 


\section{Model Validation and Improvement}

In this work, the analysis of the modulator's behavior is based on the assumption that the FLC cell can be modeled by a half-wave plate. To confirm this assumption, the estimated angle shown in Fig. 4 is backfed into Eq. (6), which represents the intensity the system would measure if the FLC cell was an ideal half-wave plate. These intensities are computed for the four input Stokes vectors, and shown as red dashed curves on Fig. 3. The experimental values fit fairly well the theoretical ones, except for Fig. $3(\mathrm{~b})$, in the range $[0 \mathrm{~V},+1 \mathrm{~V}]$. Nevertheless, because no voltage in that range is used in the DRM determination, the model can be considered as accurate for the considered voltage range.

However, the model has been slightly simplified before DRM determination. Using Eqs. (16) and (17), respectively, the evolution of the physical parameters $\theta$ and $d$ versus voltage can be described, and feeding $\theta$ and $d$ directly into Eq. (8) would be possible. Nevertheless, to minimize the model mismatch that actually occurs (the depolarization of the FLC cell is assumed to be isotropic, but it may not), a purely numerical operation is performed directly from intensity measurements, almost regardless of model: the only assumption is now that the FLC cell does not ellipticize incoming light. Clearly, referring to $\mathrm{Lu}$ and Chipman decomposition model [27], we still consider a $\pi$ retardance, like with a perfect half-wave plate, but diattenuation and depolarization matrices may be more general than those of an ideal half-wave plate. The consistency of this assumption is discussed in Subsection 4.D.. Then Eq. (므) becomes

$$
\begin{aligned}
I(V)= & s_{\text {out } 0}(V)=\frac{1}{2}\left[s_{\mathrm{in} 0} \cdot A(V)+s_{\mathrm{in} 1} \cdot B(V)\right. \\
& \left.+s_{\mathrm{in} 2} \cdot C(V)+s_{\mathrm{in} 3} \cdot D(V)\right] .
\end{aligned}
$$

The above-mentioned assumption leads to $D(V)=0$, and then the output intensities relative to the input Stokes basis are written as

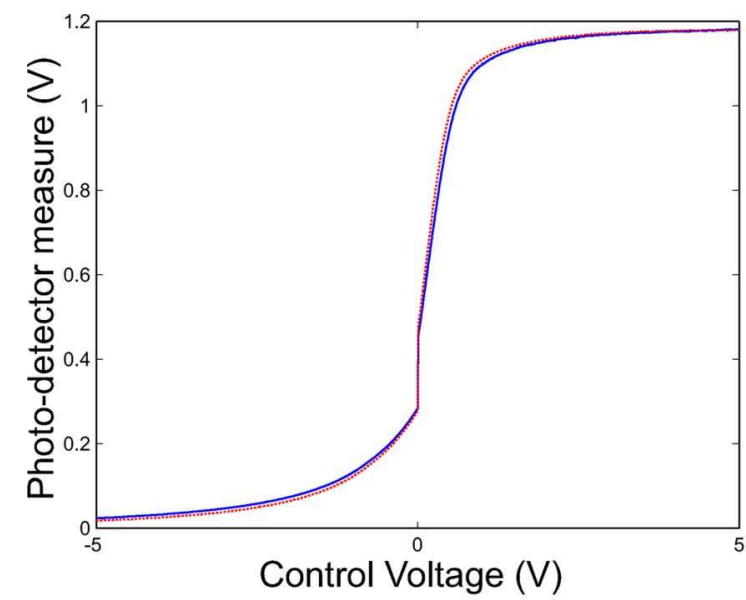

(a)

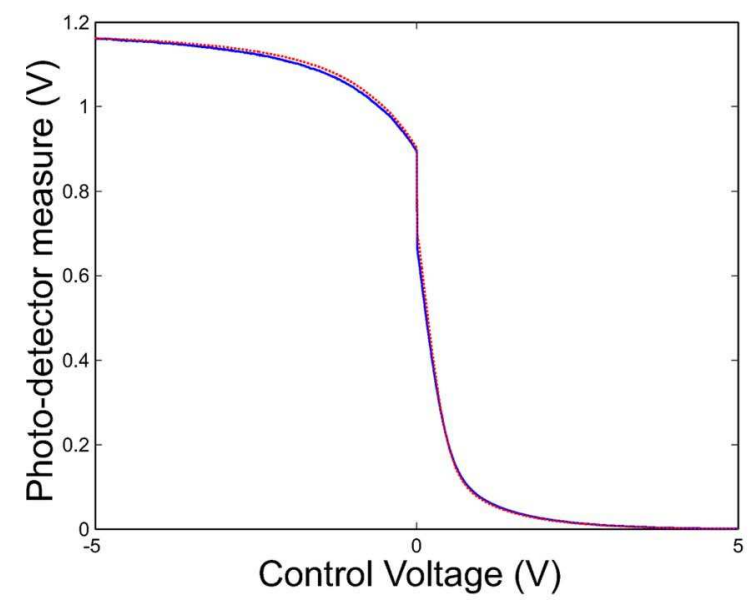

(c)

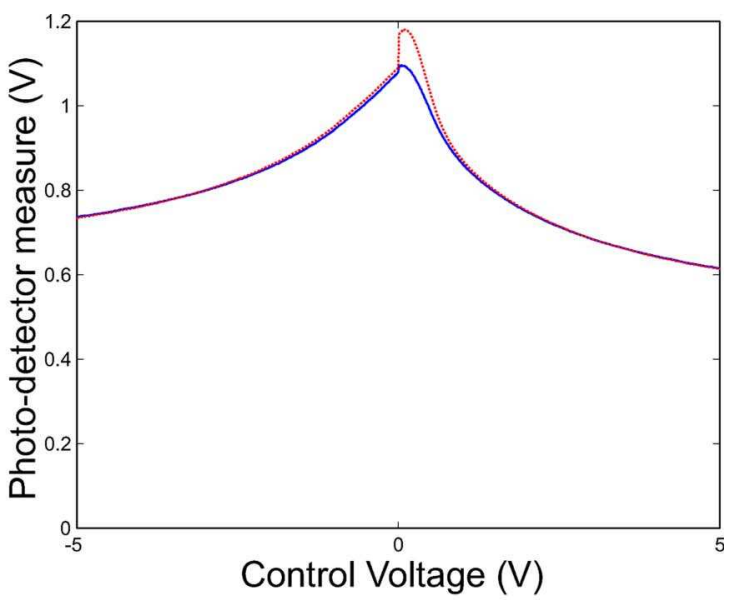

(b)

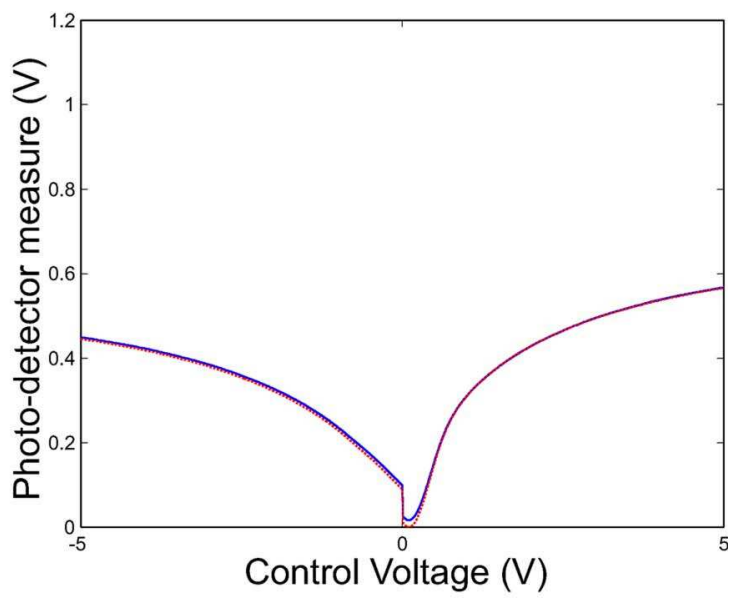

(d)

Fig. 3. (Color online) Blue continuous line: average measured intensities (a) $I_{h}$, (b) $I_{45}$, (c) $I_{v}$, and (d) $I_{135}$ of five acquisitions versus FLC control voltage for each input Stokes vectors $\mathbf{S}_{\mathbf{h}}, \mathbf{S}_{\mathbf{4 5}}, \mathbf{S}_{\mathbf{v}}$, and $\mathbf{S}_{\mathbf{1 3 5}}$. Red dashed line: intensities theoretically measured with an ideal halfwave plate whose orientation varies according to Fig. $\underline{4}$. 


$$
\left\{\begin{array}{cc}
I_{h}(V)=\frac{1}{2}[A(V)+B(V)] & \text { for } \mathbf{S}_{\mathbf{h}} \text { in input } \\
I_{v}(V)=\frac{1}{2}[A(V)-B(V)] & \text { for } \mathbf{S}_{\mathbf{v}} \text { in input } \\
I_{45}(V)=\frac{1}{2}[A(V)+C(V)] & \text { for } \mathbf{S}_{\mathbf{4 5}} \text { in input } \\
I_{135}(V)=\frac{1}{2}[A(V)-C(V)] & \text { for } \mathbf{S}_{\mathbf{1 3 5}} \text { in input }
\end{array}\right.
$$

responses for the $\mathbf{S}_{\mathbf{h}}$ Stokes vector input are presented in Figs. 6(c) and 6(d), respectively, for the amplitudes of 5 and $0.1 \mathrm{~V}$, for both positive and negative control voltages. We verify that the modulator response reaches a steady state at any of the four voltages (time responses for other polarization inputs are not reported because they are rather similar).

The system matrix can therefore be expressed as

$$
\mathbf{A}=\frac{1}{2}\left[\begin{array}{lll}
\frac{I_{h}\left(V_{1}\right)+I_{v}\left(V_{1}\right)+I_{45}\left(V_{1}\right)+I_{135}\left(V_{1}\right)}{I_{h}\left(V_{2}\right)+I_{v}\left(V_{2}\right)+I_{45}\left(V_{2}\right)+I_{135}\left(V_{2}\right)} & I_{h}\left(V_{1}\right)-I_{v}\left(V_{1}\right) & I_{45}\left(V_{1}\right)-I_{135}\left(V_{1}\right) \\
\frac{I_{h}\left(V_{3}\right)+I_{v}\left(V_{3}\right)+I_{45}\left(V_{3}\right)+I_{135}\left(V_{3}\right)}{2} & I_{h}\left(V_{3}\right)-I_{v}\left(V_{3}\right) & I_{45}\left(V_{2}\right)-I_{135}\left(V_{2}\right) \\
\frac{\left.I_{3}\right)-I_{135}\left(V_{3}\right)}{2}
\end{array}\right]
$$

\section{Control Signal Optimization}

With the three chosen voltages, a composite control signal with four levels $\{+5 \mathrm{~V},-5 \mathrm{~V},+0.1 \mathrm{~V},-0.1 \mathrm{~V}\}$ is designed because the modulator requires a DCbalanced input signal to avoid damage. It is presented in Fig. 6(b). Each voltage level corresponds to a specific state of the liquid crystals, and the pulse aims to reduce the transition time between two successive states. Because each voltage is applied over $5 \mathrm{~ms}$, three useful acquisitions can be performed every $20 \mathrm{~ms}$. Therefore, the polarization is analyzed at an average frequency of $150 \mathrm{~Hz}$. The modulator is characterized as previously, except that the FLC cell is driven with the composite control signal instead of the bipolar signal used during the previous characterization, Fig. 6(a). The bipolar control signal corresponds to the native control signal of the device. Time

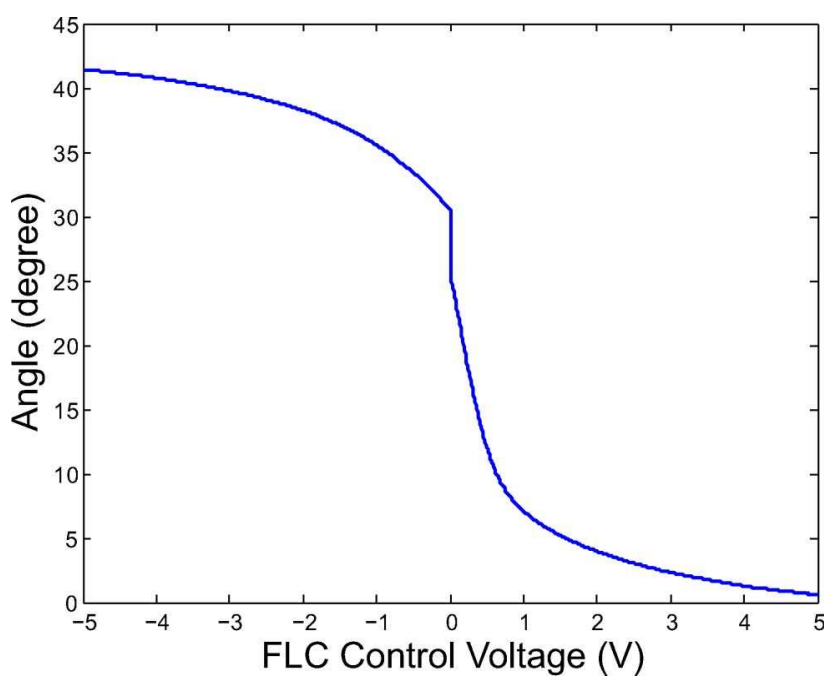

Fig. 4. (Color online) FLC orientation angle estimation (average of five acquisitions).
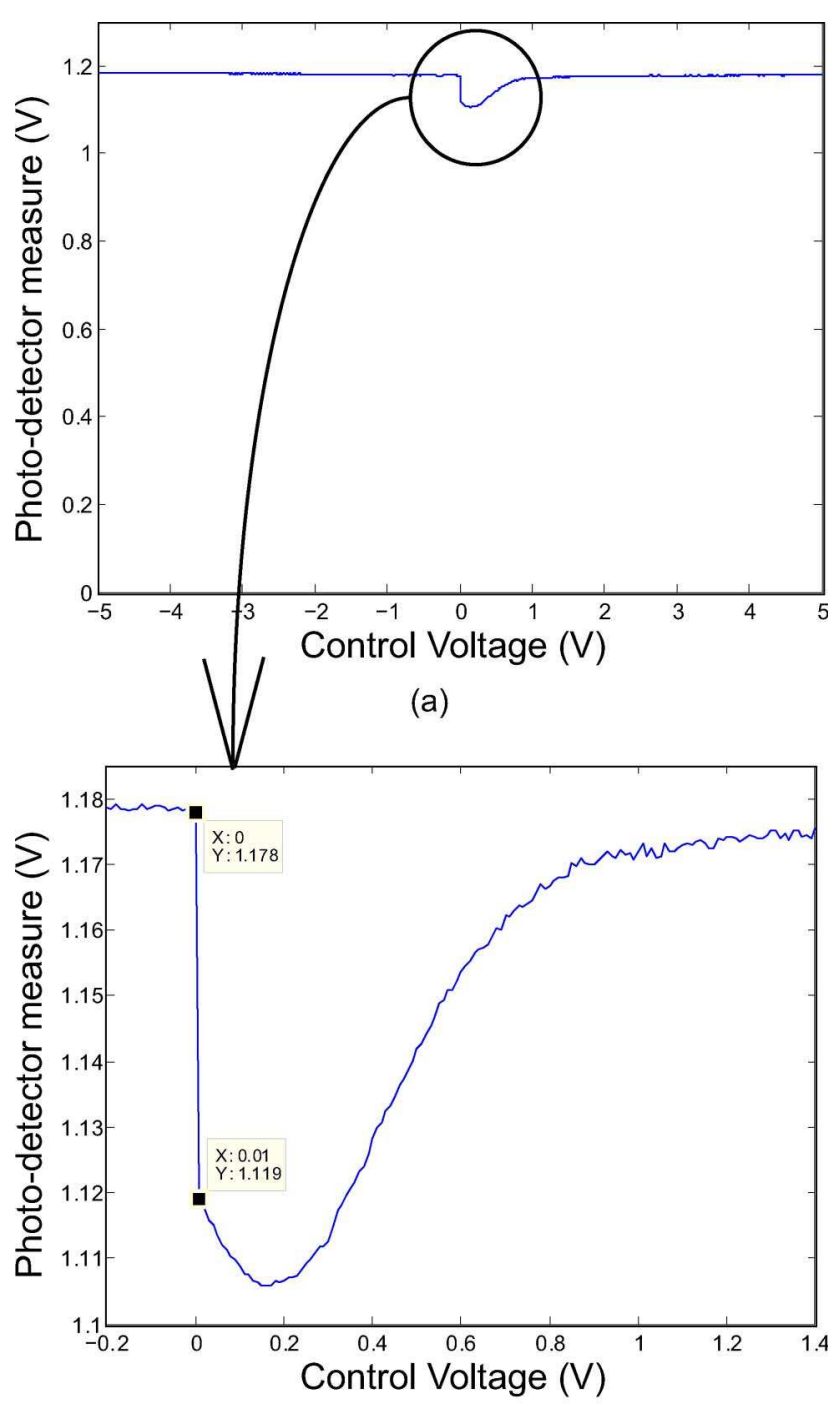

(b)

Fig. 5. (Color online) Variation of intensity (a) $I_{h}+I_{v}$ and (b) zoom at a specific voltage range of the curve $I_{h}+I_{v}$. 
Figures 6(c) and 6(d) clearly show that the time responses are different with bipolar and composite control signals. Either the steady-state values differ, or the whole shapes of the time responses differ. Actually, the modulator state depends on both the voltage currently applied on the FLC cell and the voltage previously applied. Therefore, a complete characterization of the modulator using the composite signal instead of separate bipolar signals should be performed. It should be noted that changing the order of the voltages in the composite control signal without changing the voltages themselves might result in a different modulator characteristic. Although this is an area for improvement, this point is not be discussed in this paper.

Finally, the complete characterization of the system using the composite control signal leads to the experimental DRM:

$$
\mathbf{A}^{-1}=\left[\begin{array}{ccc}
0.7743 & -0.2453 & 0.6094 \\
-0.7140 & 0.1844 & 0.5375 \\
-1.4477 & 1.8313 & -0.3789
\end{array}\right]
$$

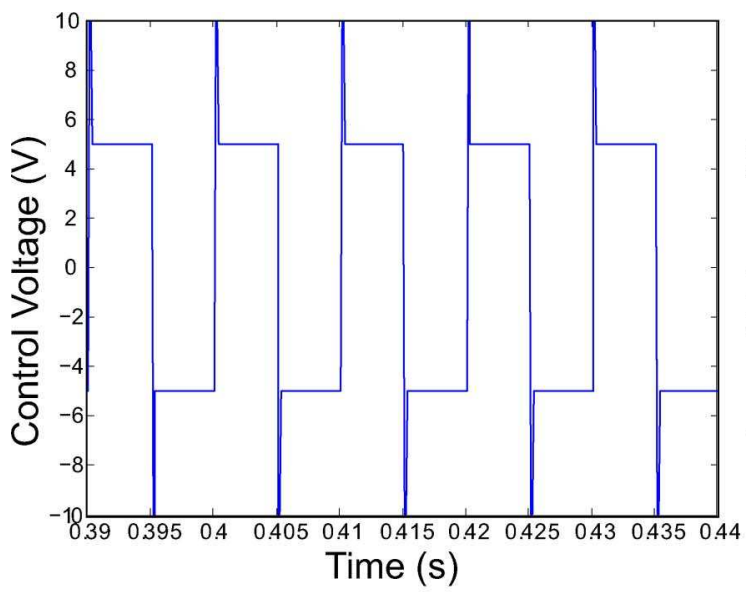

(a)

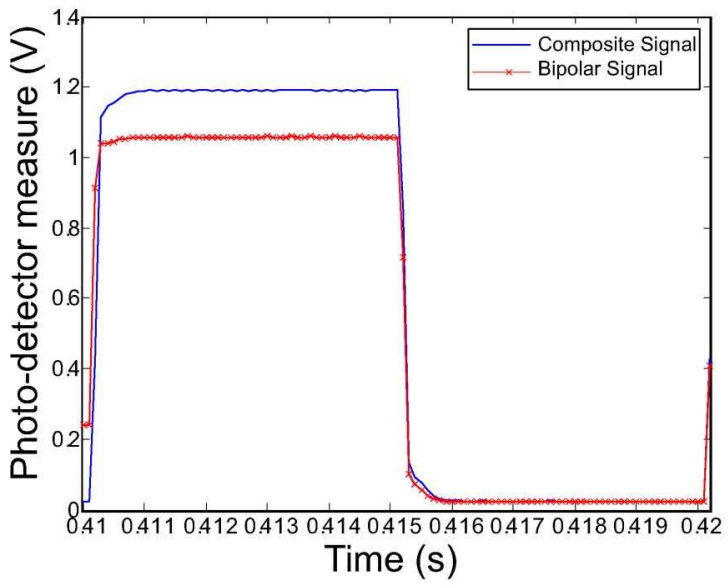

(c)
Using an ideal half-wave plate model with angles $\left\{\theta_{i}\right\}=\left\{45^{\circ}, 22.5^{\circ}, 0^{\circ}\right\}$ would have led to

$$
\mathbf{A}^{-1}=\left[\begin{array}{ccc}
1 & 0 & 1 \\
-1 & 0 & 1 \\
-1 & 2 & -1
\end{array}\right]
$$

The difference between the ideal half-wave plate model and the depolarizing half-wave plate model has been evaluated considering the system condition number $(\mathrm{CN})$, which is relative to the system ability to be inverted and should be minimized. The result is presented in Fig. 7. For an ideal half-wave plate rotating from $0^{\circ}$ to $180^{\circ}$, it has been shown that the configuration leading to the optimal CN (value $\sqrt{2}$ ) was the equally spaced configuration $\left\{\theta_{i}\right\}=\left\{0^{\circ}, 60^{\circ}, 120^{\circ}\right\}$ [26]. In our case, the azimuthal angle of the equivalent half-wave plate is limited to $\left[0^{\circ}, 45^{\circ}\right]$. For extremal angles $\left\{0^{\circ}, 45^{\circ}\right\}$, the minimum condition number is obtained with $\left\{\theta_{i}\right\}=\left\{0^{\circ}, 22.5^{\circ}, 45^{\circ}\right\}$, leading to an ideal $\mathrm{CN}$ value around 2.41. For the above-described experimental system with extremal angles $\left\{0.5^{\circ}, 41.5^{\circ}\right\}$, the minimum condition number value is 2.98 and is obtained with $\left\{\theta_{i}\right\}=\left\{0.5^{\circ}\right.$,

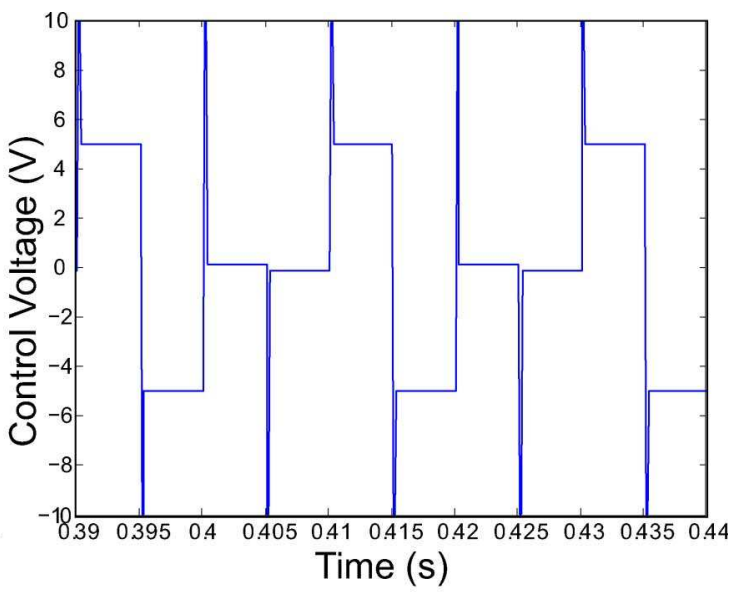

(b)

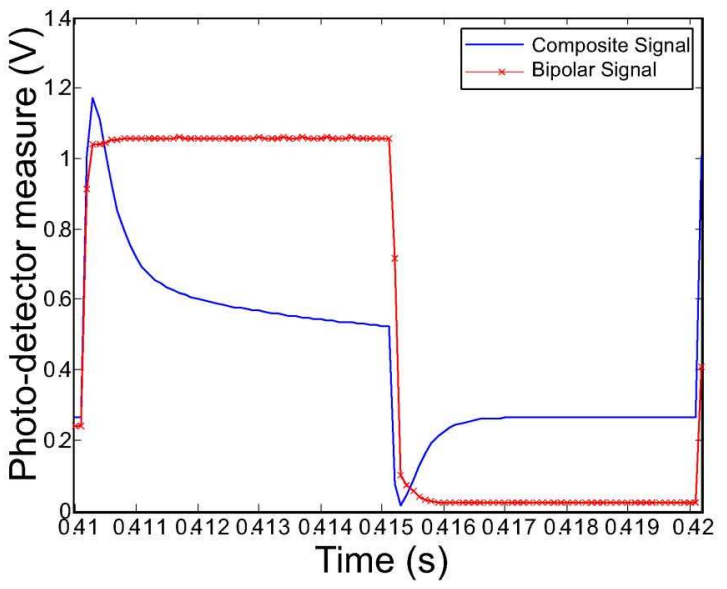

(d)

Fig. 6. (Color online) (a) Bipolar control signal, (b) composite control signal, (c) FLC cell responses to bipolar and composite signals at specific amplitudes of $5 \mathrm{~V}$, and (d) $0.1 \mathrm{~V}$ for $\mathbf{S}_{\mathbf{h}}$ polarization input. 


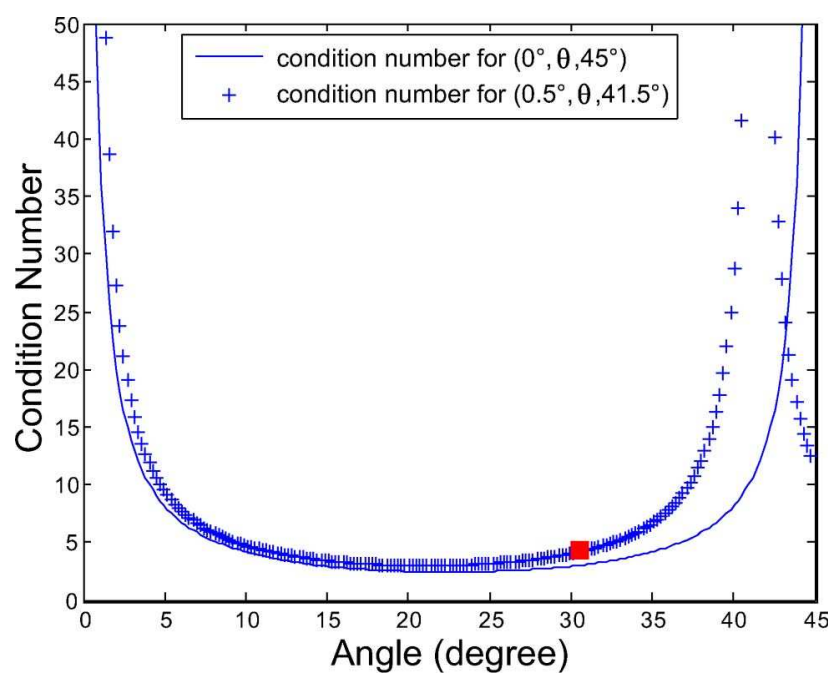

Fig. 7. (Color online) Condition number for ideal and experimental systems. The red square denotes our actual configuration.

$\left.20.9^{\circ}, 41.5^{\circ}\right\}$. The intermediate angle $20.9^{\circ}$ is rather far from the angle we chose $\left(31.2^{\circ}\right)$. This does not seem too critical because the $\mathrm{CN}$ value for $\left\{\theta_{i}\right\}=$ $\left\{0.5^{\circ}, 31.2^{\circ}, 41.5^{\circ}\right\}$ is 4.36 , which is rather low provided the intermediate angle is far enough (let us say $5^{\circ}$ ) from the extremal values.

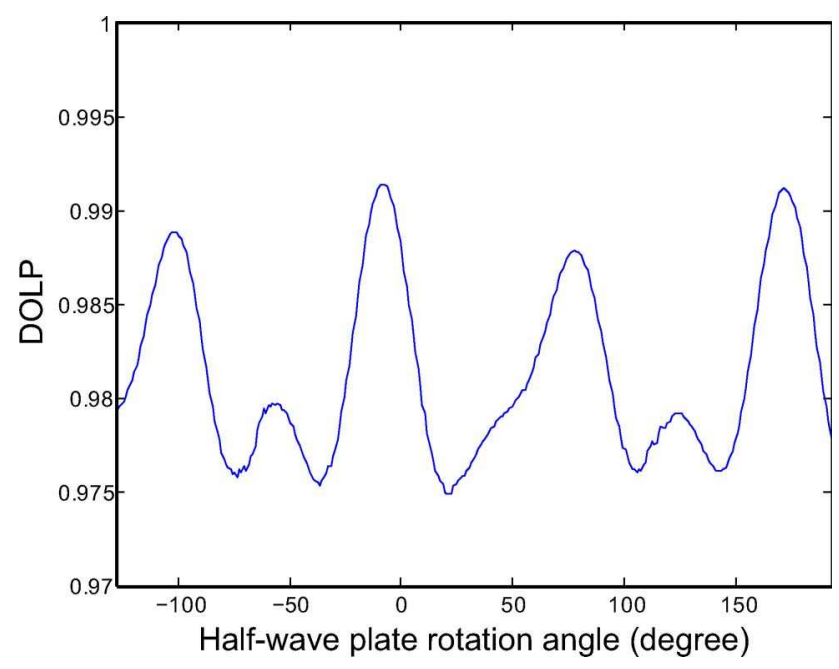

Fig. 9. (Color online) Estimation of the DOLP of the light emerging from a rotating half-wave plate.

\section{Experimental Validation}

\section{A. One-Dimensional Validation}

The bench used for this validation is the same as the one used for the characterization, Fig. 2. Here, the modulator is driven by the composite control signal and the half-wave plate rotates to generate different

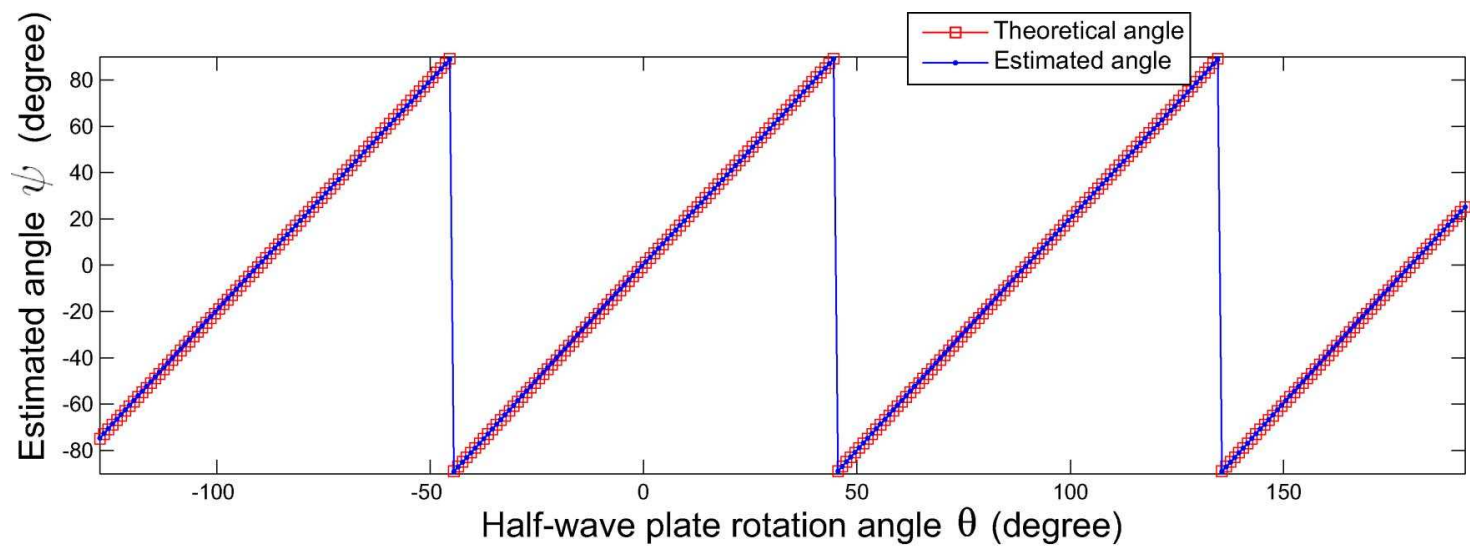

(a)

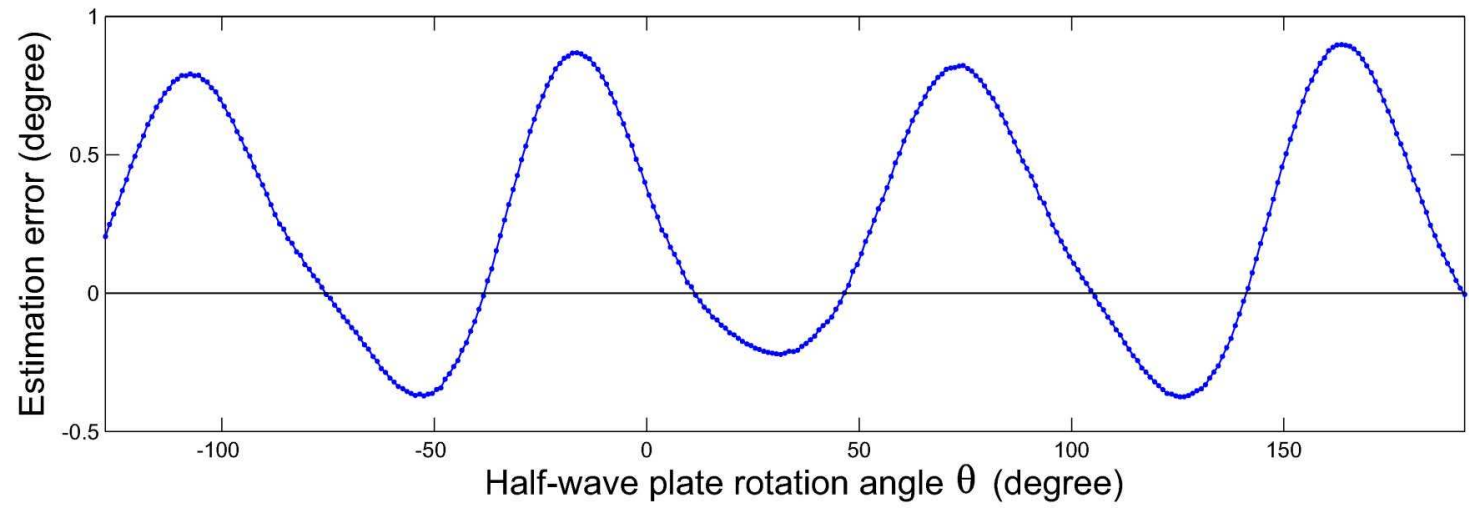

(b)

Fig. 8. (Color online) (a) Estimated angle in practice and theoretical angle of the polarization of the light emerging from a rotating half-wave plate. (b) Angle estimation error. 


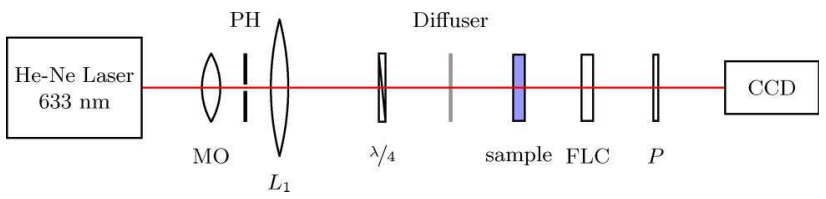

Fig. 10. (Color online) Setup for imaging validation: microscope objective (MO), pinhole $(\mathrm{PH})$, lens $\left(L_{1}\right)$ quarter-wave plate $(\lambda / 4)$, ferroelectric liquid crystal cell (FLC), static linear polarizers oriented at $0^{\circ}(P)$.

polarization states that are analyzed by the modulator. These polarization states include $\mathbf{S}_{\mathbf{h}}, \mathbf{S}_{\mathbf{v}}, \mathbf{S}_{45}$, and $\mathbf{S}_{\mathbf{1 3 5}}$, used for characterization, but are not limited to them. The results are presented Figs. 8 and 9 .

Figure $8(\mathrm{a})$ shows the estimation of the polarization angle $\psi$ with respect to the rotating half-wave plate angle $\theta$. We observe that the two curves fit each other. The estimation precision is described more precisely by Fig. 8(b), which represents the estimation error computed as the signed difference between the estimated angle and the theoretical angle. The error varies around a mean of $0.25^{\circ}$, with a variation of $1.27^{\circ}$ between maximum and minimum values. We consider this precision as fairly good, although we did not find in the literature a numeric evaluation of precision to compare this number to. Oscillations in the error curve may be explained by a wobble in the rotation of the half-wave plate or a defect in the half-wave plate geometry itself.

The estimation of the DOLP of the incident Stokes vector estimated by the PSA is presented in Fig. 9 . If the incident polarization is indeed totally linearly polarized, a DOLP constant equal to 1 is expected. In practice, the DOLP varies between 0.991 and 0.975. Possible explanations for this phenomenon might be an estimation error due to a nonoptimal

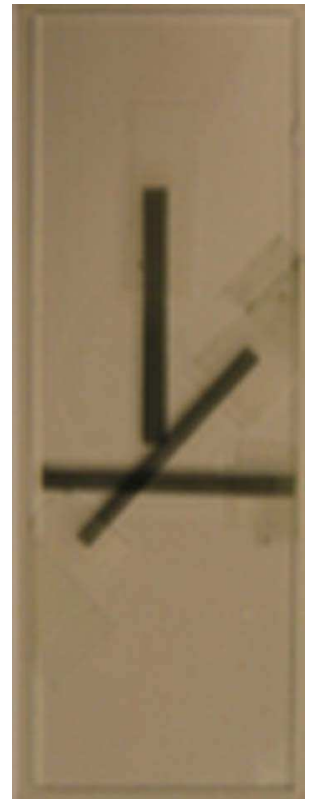

Fig. 11. (Color online) Test sample used for imaging validation: a microscope plate with three strips of linear polarizer.
DRM, a wobble in the rotation of the half-wave plate, or a defect in the half-wave plate geometry itself.

\section{B. Two-Dimensional Validation}

The setup used for imaging validation, presented in Fig. 10, is rather similar to that used for the characterization. An additional quarter-wave plate $(\lambda / 4)$ is used to produce a circularly polarized input light. The use of a rotating diffuser between the quarter-wave plate and the observed sample aims at reducing the speckle noise. The sample is followed by the modulator (FLC, $P$ ) which is driven by the composite control signal. The photodetector used previously is replaced by a CCD video camera.

The test sample is composed of a microscope plate on which are fixed three strips of linear polarizers oriented at $0^{\circ}, 45^{\circ}$, and $90^{\circ}$ (Fig. 11). For technical reasons, the analysis direction of polarizer $P$, which was set to be the horizontal direction previously, is not a remarkable direction in this section. Actually, for the sake of clarity, the test sample is oriented so

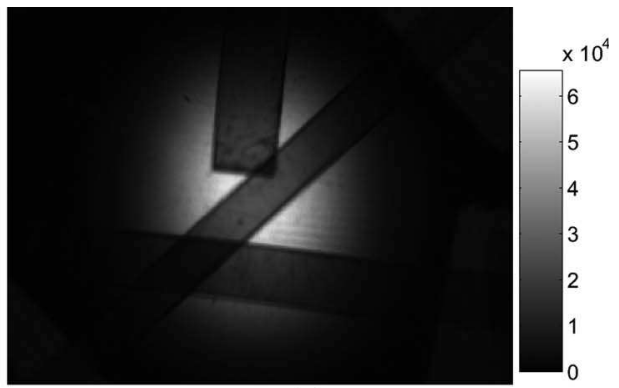

(a)

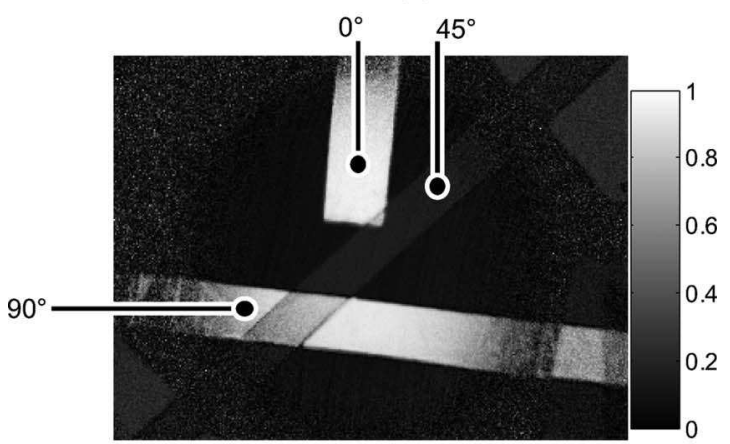

(b)

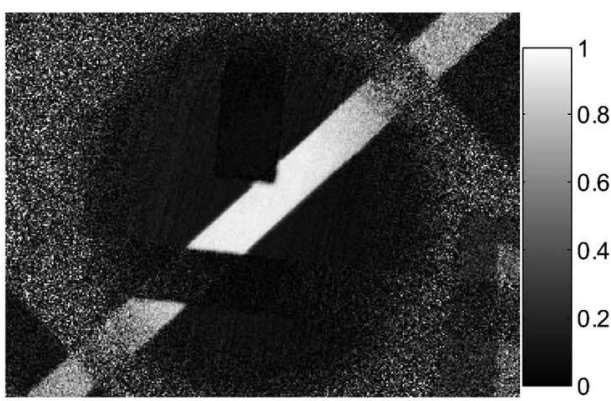

(c)

Fig. 12. Stokes vector estimation, at a frame rate of $150 \mathrm{fps}$, for a test sample oriented at $0^{\circ}$. (a) $s_{0}$, (b) $\left|s_{1}\right| / s_{0}$, and (c) $\left|s_{2}\right| / s_{0}$. Polarizer orientations are mentioned in (b). 
that one polarizer strip is aligned with the analysis direction, and this position is set to be the angular reference at $0^{\circ}$. The sample is mounted on a rotation stage, but it is not studied in motion. Once the object is oriented at a specific angle, 100 frames of Stokes vector, angle, and DOLP are estimated. Polarization information is available at $150 \mathrm{fps}$. The image is centered on the three polarizer strips, with $320 \times 240$ pictures and 14 bits per pixel. Stokes vector, angle, and DOLP estimations at different rotation angles of the test sample are shown in Figs. 12-14.

Qualitatively speaking, Stokes estimations presented in Fig. 12 are fairly good. As expected, polarizers oriented at $0^{\circ}$ and $90^{\circ}$ appear bright on the $s_{1}$ component, while the polarizer at $45^{\circ}$ is dark. The contrary is observable on the $s_{2}$ component.

Angle estimations (Fig. 13) also match expected values because the three polarizers are oriented at $45^{\circ}$ one from another. Sides of pictures appear noisy because light intensity is low in these parts due to the beam shape. In the center of the pictures, on areas around the polarizers, a constant polarization angle is estimated for the background, whereas the background light is supposed to be circularly polarized. This shows that a linearly polarized component remains because of calibration precision or plate defects. Anyway, angle figures should not be analyzed without DOLP figures because they may not be related to a very consistent linearly polarized component. On DOLP pictures, the background is dark, which means that the remaining linear component is weak. On the contrary, polarizer strips appear bright, highly linearly polarized.

To evaluate quantitative results, regions of interest (ROI) of 459 pixels are arbitrarily defined on each polarizer strip, as presented in Fig. 15. On each ROI, and for each of the 100 available frames, spatially averaged data are evaluated. Figure 16 shows quantitative results versus frame index, which is the temporal evolution of the data, for a sample oriented at $0^{\circ}$, as depicted in Fig. 14(a).

First, error depends on the orientation of the polarizers. The spatially averaged angle error can reach up to $2.75^{\circ}$, with a temporal mean around $2.1^{\circ}$, which is more than what was obtained for the one-dimensional validation. A similar observation can be made for the DOLP, in which the error reaches 0.09 for one of the polarizer strips. Even if a part of the angular error might be attributed to a bad angular reference, a major part of the error for both the angle and the DOLP can be attributed to noise. In Fig. 14, polarizers appear more or less noisy depending on the scene orientation. This noise is partly introduced by the diffuser, composed of a rotating disk, responsible for an rms of $3.5 \%$ light intensity variation for a $1.5 \%$ depolarization. A few simulations showed that such a variation was responsible for the dramatic loss in precision on the angle and the DOLP. In addition, the implementation of the diffuser does not affect the scene uniformly, adding noise on one component more than the other. Extra experiments showed that, in this

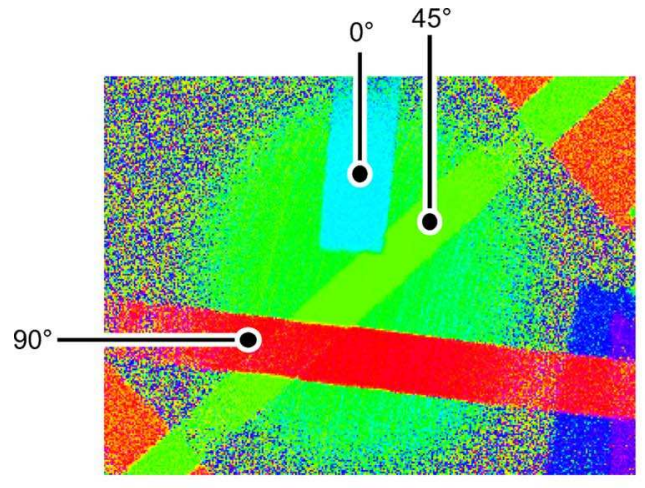

(a)

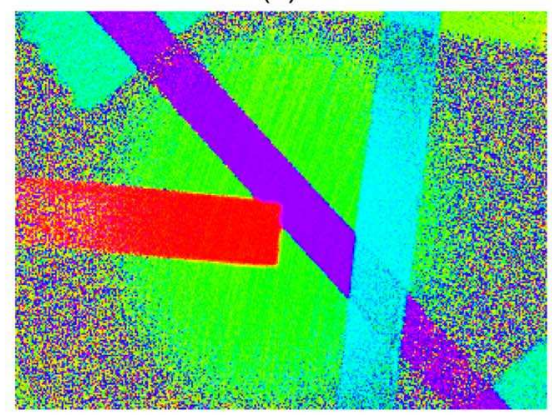

(c)

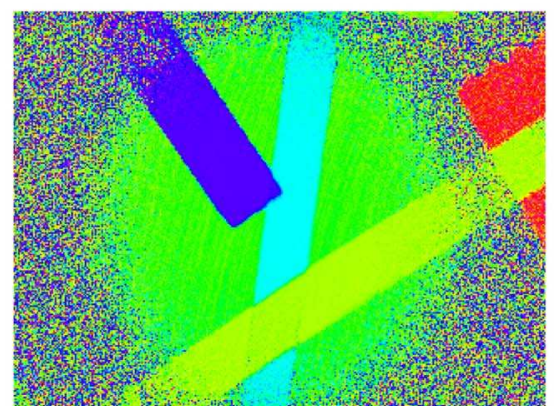

(b)

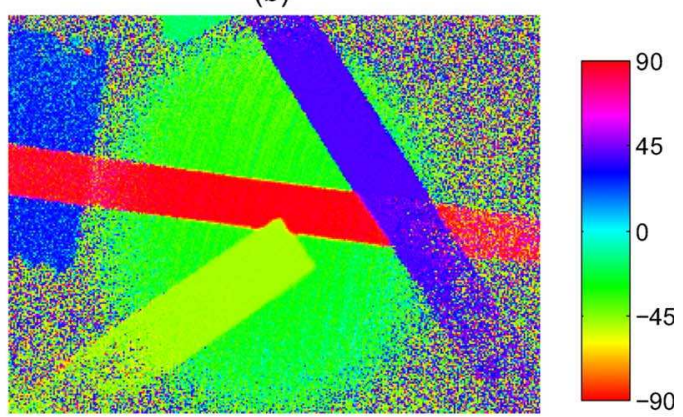

(d) (e)

Fig. 13. (Color online) Angle estimation, at a frame rate of $150 \mathrm{fps}$, for different rotation angles of the test sample: (a) $0^{\circ}$, (b) $40^{\circ}$, (c) $90^{\circ}$, and (d) $130^{\circ}$. (e) Color bar. The reference position at $0^{\circ}$ is arbitrarily defined so that one polarizer strip is actually aligned with the modulator axis. Polarizers orientations are mentioned in (a). 


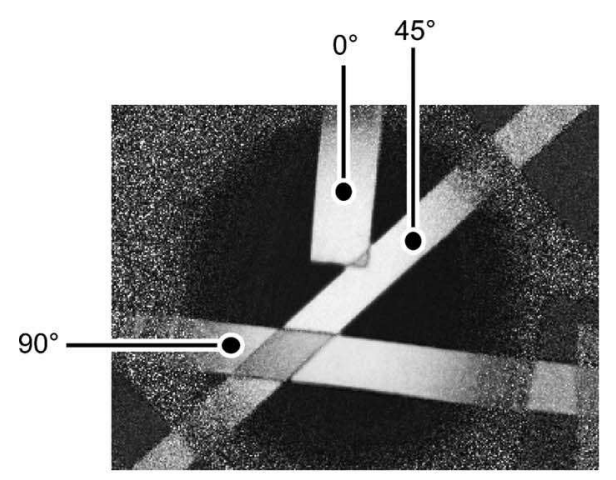

(a)

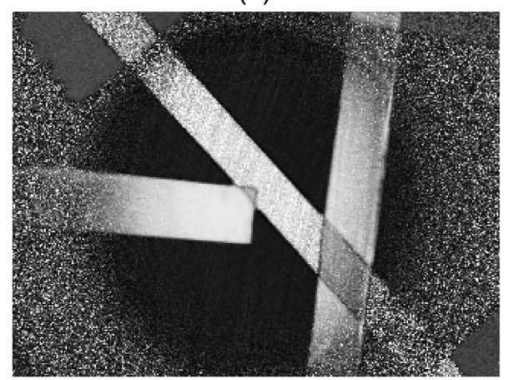

(c)

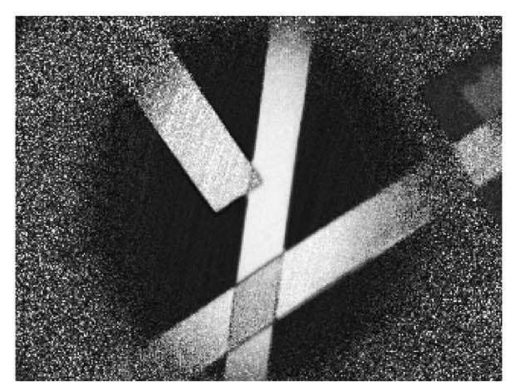

(b)

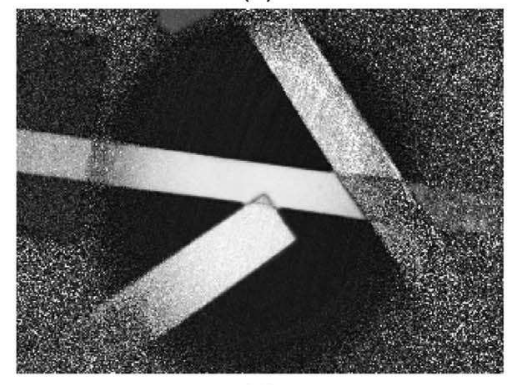

(d)

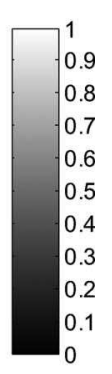

(e)

Fig. 14. DOLP estimation, at a frame rate of $150 \mathrm{fps}$, for different rotation angles of the test sample: (a) $0^{\circ}$, (b) $40^{\circ}$, (c) $90^{\circ}$, and (d) $130^{\circ}$. (e) Color bar. Polarizers orientations are mentioned in (a).

case, performing 8 bit acquisitions instead of 14 bit acquisitions only provides very little degradation.

Moreover, the intensity of the light incident on the object also influences noise. For example, in Fig. 14(d), the polarizer strip at the top right is at the edge of the gaussian light beam, where light is lower than in the center. This polarizer exhibits a DOLP smaller than the other polarizers, rather noisy, whereas in Fig. 14(a), this polarizer, which was almost horizontal, appears with a high DOLP, with very little noise.

\section{Discussion and Possible Improvements}

Improving the system accuracy is possible in three main ways. A first way of improvement could be an experimental study of the influence of the voltage order in the composite signal on DRM conditioning

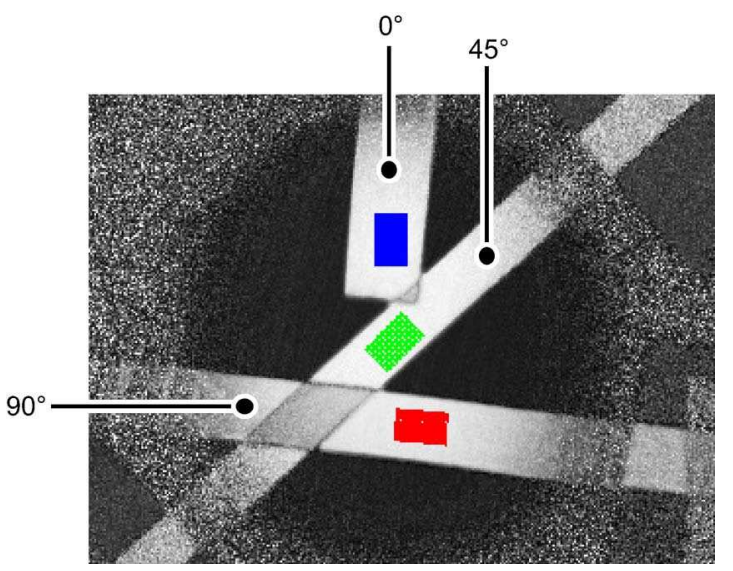

Fig. 15. (Color online) Regions of interest used to quantify the results, presented on a DOLP picture of a test sample oriented at $0^{\circ}$. Polarizers orientations are also mentioned. because the modulator did not prove to be a timeinvariant linear response system, as mentioned in Subsection 4.D.

Another way would consist of deeply studying the modulator response in the range $[0 \mathrm{~V},+1 \mathrm{~V}]$, where the model used for the FLC cell did not correspond to the actual modulator's behavior. Using this voltage range was avoided in the present work. Nevertheless, should a more general model of waveplate for the FLC cell be considered, i.e., taking account of the circularity produced by the modulator, this voltage range could probably be used with much benefit.

It would also make possible another improvement. In the present work, the composite control signal consists of four different voltages (pulse apart): $+5 \mathrm{~V}$, $-5 \mathrm{~V},+0.1 \mathrm{~V}$, and $-0.1 \mathrm{~V}$, since in a first approach an even length sequence is the simplest way to produce a DC-balanced signal. The polarization state corresponding to $+0.1 \mathrm{~V}$ is currently ignored. If the voltage range $[0 \mathrm{~V},+1 \mathrm{~V}]$ could be used with confidence, this fourth response could be taken into account and then, at no additional cost, a $4 \times 4 \mathrm{DRM}$ could be considered and noise influence could be lowered.

Improving the system speed can also be considered. It can classically consist of running the modulator and the camera at a higher frame rate [28], since this FLC cell was successfully operated in bistable mode at above $2 \mathrm{kHz}$. Another way consists, as mentioned earlier, in considering the response of the fourth voltage control, provided it is accurately modeled. Because, in the current design, the voltage is sent to the modulator, even if its response is not considered, it would not slow down the system but, on 


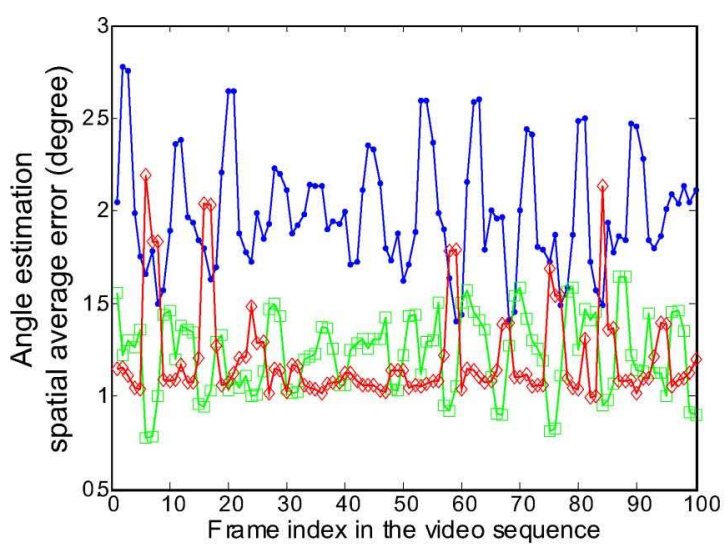

(a)

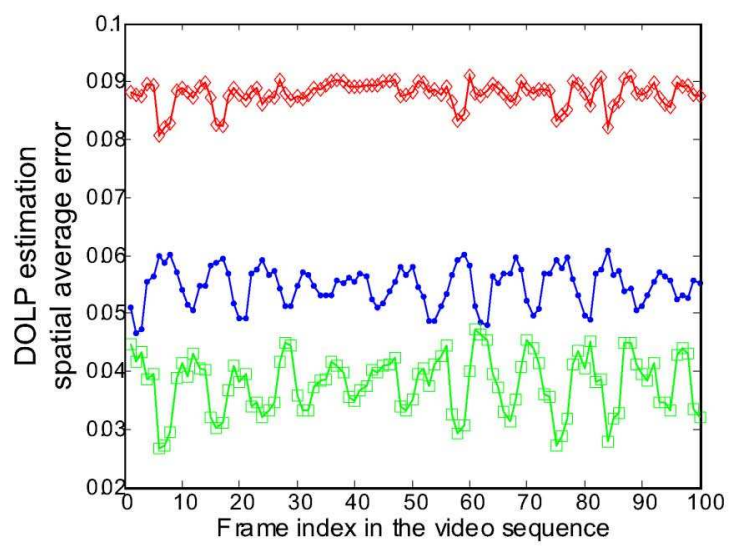

(c)

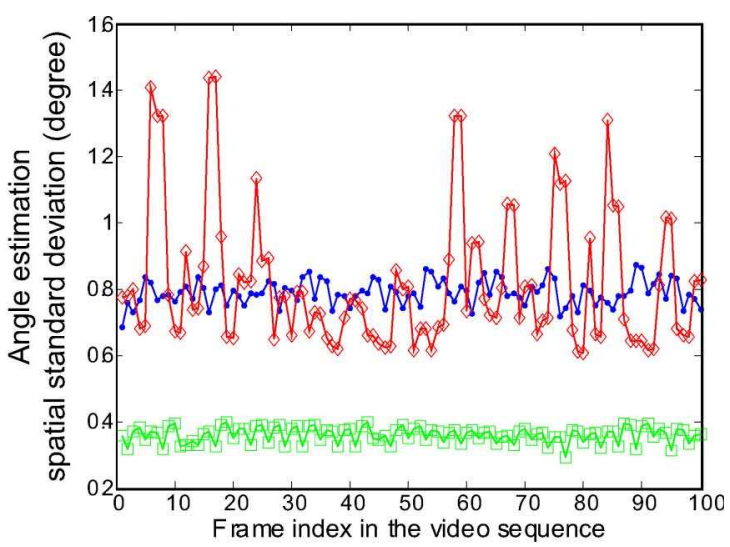

(b)

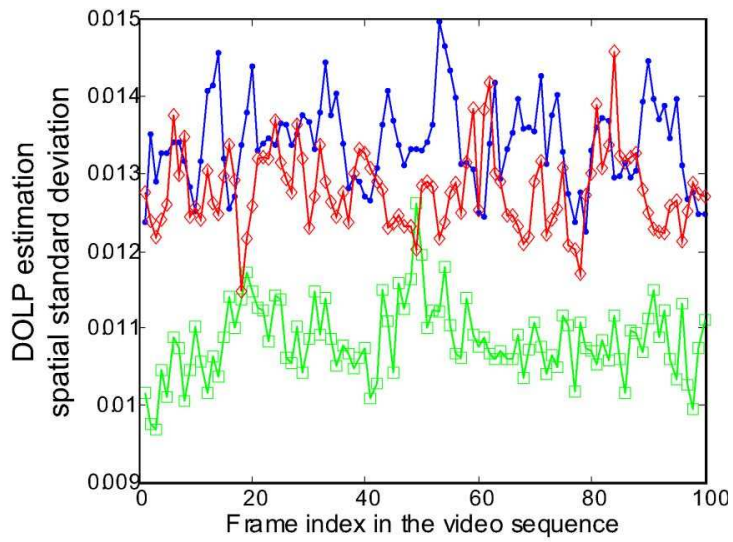

(d)

Fig. 16. (Color online) Spatial average error and standard deviation of the angle and DOLP estimation, at a frame rate of 150 fps, for a test sample oriented at $0^{\circ}$. Blue line with dots: polarizer strip oriented at $0^{\circ}$. Green line with squares: polarizer strip oriented at $45^{\circ}$. Red line with diamonds: polarizer strip oriented at $90^{\circ}$.

the contrary, make consistent polarimetric information available at every polarization state (instead of three polarization states out of four), which would increase the frame rate from 150 to $200 \mathrm{fps}$ at no additional cost.

\section{Conclusion}

A lightweight portable imaging polarimeter using a single ferroelectric liquid crystal cell was designed and implemented. It was demonstrated to capture full linear polarimetric information (i.e., the first three Stokes parameters) at $150 \mathrm{fps}$. Whereas mostly used as bistable components until now, ferroelectric liquid crystal cells can also be used as tunable plates at their nominal wavelength with much benefit, as shown in this paper. For a wide range of input voltages, their behavior is close to that of half-wave plates whose orientation is tunable. Such a simple optical design paves the way for new applications where high-speed and portable implementations are required.

Polarization state analyzer performance was deeply studied through nonimaging experiments, showing an average precision of $2 \%$ on the degree of linear polarization and $0.25^{\circ}$ on azimuthal angle. Imaging experiments were also successfully conducted at $150 \mathrm{fps}$. Improvements consisting in optimizing the LC cell control could slightly enhance the system performance.

\section{References}

1. J. S. Tyo, D. Goldstein, D. Chenault, and J. A. Shaw, "Review of passive imaging polarimetry for remote sensing applications," Appl. Opt. 45, 5453-5469 (2006).

2. D. A. Lavigne, M. Breton, M. Pichette, V. Larochelle, and J.-R. Simard, "Evaluation of active and passive polarimetric electro-optic imagery for civilian and military targets discrimination," Proc. SPIE 6972, 69720X (2008).

3. A. M. Gandorfer, "Ferroelectric retarders as an alternative to piezoelastic modulators for use in solar stokes vector polarimetry," Opt. Eng. 38, 1402-1408 (1999).

4. J. M. Bueno and P. Artal, "Double-pass imaging polarimetry in the human eye," Opt. Lett. 24, 64-66 (1999).

5. G. Stokes, "On the composition and resolution of streams of polarized light from different sources," Trans. Camb. Philos. Soc. 9, 339-416 (1852).

6. J. Solomon, "Polarization imaging," Appl. Opt. 20, 1537-1544 (1981).

7. E. de Leon, R. Brandt, A. Phenis, and M. Virgen, "Initial results of a simultaneous stokes imaging polarimeter," Proc. SPIE 6682, 668215 (2007). 
8. K. Fujita, Y. Itoh, and T. Mukai, "Development of simultaneous imaging polarimeter for asteroids," Adv. Space. Res. 43, 325-327 (2009).

9. J. S. Harchanko, L. Pezzaniti, D. Chenault, and G. Eades, "Comparing a MWIR and LWIR polarimetric imager for surface swimmer detection," Proc. SPIE 6972, 697211 (2008).

10. F. A. Sadjadi and C. S. L. Chun, "Remote sensing using passive infrared stokes parameters,” Opt. Eng. 43, 2283-2291 (2004).

11. A. Gruev, A. Ortu, N. Lazarus, J. van der Spiegel, and N. Engheta, "Fabrication of a dual-tier thin film micropolarization array," Opt. Express 15, 4994-5007 (2007).

12. R. Walraven, "Polarization imagery," Proc. SPIE 112, 164-167 (1977).

13. L. B. Wolff, T. A. Mancini, P. Pouliquen, and A. G. Andreou, "Liquid crystal polarization camera," IEEE Trans. Robot. Autom. 13, 195-203 (1997).

14. A. Jaulin and L. Bigué, "High speed linear polarization evaluation with a single light modulator," in EOS 3rd Topical Meeting on Advanced Imaging Techniques (European Optical Society, 2007).

15. A. Jaulin, L. Bigué, and P. Ambs, "High-speed degree-ofpolarization imaging with a ferroelectric liquid-crystal modulator," Opt. Eng. 47, 033201 (2008).

16. D. Goldstein, Polarized Light, 2nd ed. (Marcel Dekker, 2003).

17. R. A. Chipman, "Depolarization index and the average degree of polarization," Appl. Opt. 44, 2490-2495 (2005).

18. M. Alouini, F. Goudail, P. Réfrégier, A. Grisard, E. Lallier, and D. Dolfi, "Multispectral polarimetric imaging with coherent il- lumination: towards higher image contrast," Proc. SPIE 5432, 133-144 (2004).

19. M. W. Williams, "Depolarization and cross polarization in ellipsometry of rough surfaces," Appl. Opt. 25, 3616-3622 (1986).

20. P. Clémenceau, A. Dogariu, and J. Stryewski, "Polarization active imaging," Proc. SPIE 4035, 401-409 (2000).

21. URL:http://www.bnonlinear.com/products/polarRotators/ polarRotators.htm.

22. A. Jaulin and L. Bigué, "High speed partial stokes imaging using a ferroelectric liquid crystal modulator," J. Eur. Opt. Soc. Rap. Pub. 3, 08019 (2008).

23. R. A. Chipman, "Polarimetry," in Handbook of Optics, M. Bass, ed. (McGraw-Hill, 1995).

24. D. S. Sabatke, M. R. Descour, E. L. Dereniak, W. C. Sweatt, S. A. Kemme, and G. S. Phipps, "Optimization of retardance for a complete stokes polarimeter," Opt. Lett. 25, 802-804 (2000).

25. K.-F. Reinhart, L. Dorfmüller, K. Marx, and T. Matszczyk, "Addressing of ferroelectric liquid crystal matrices and electrooptical characterization," Ferroelectrics 113, 405-417 (1991).

26. J. S. Tyo, "Optimum linear combination strategy for an n-channel polarization-sensitive vision or imaging system," J. Opt. Soc. Am. A 15, 359-366 (1998).

27. S.-Y. Lu and R. A. Chipman, "Interpretation of Mueller matrices based on polar decomposition,” J. Opt. Soc. Am. A 13, 1106-1113 (1996).

28. L. Bigué and N. Cheney, "High-speed portable polarimeter using a ferroelectric liquid crystal modulator," Proc. SPIE 6682, 668205 (2007). 